\title{
Lunar Eclipses and Allais Effect
}

\author{
Russell Bagdoo \\ Saint-Bruno-de-Montarville, Quebec, Canada \\ Email: rbagdoo@gmail.com
}

How to cite this paper: Bagdoo, R. (2021) Lunar Eclipses and Allais Effect. Journal of Modern Physics, 12, 1783-1794. https://doi.org/10.4236/jmp.2021.1213104

Received: September 8, 2021

Accepted: November 16, 2021

Published: November 19, 2021

Copyright (c) 2021 by author(s) and Scientific Research Publishing Inc. This work is licensed under the Creative Commons Attribution International License (CC BY 4.0).

http://creativecommons.org/licenses/by/4.0/

\begin{abstract}
Two anomalies observed during lunar eclipses, the enlargement of the Earth's shadow and the excessive clarity of the penumbra, possibly attributed to insufficient causes if not doubtful, would refute the assertion of certain experimenters according to which the lunar Allais eclipse effect would be almost impossible to detect. The Earth's umbra seems to be $2 \%$ larger than what is expected from geometrical considerations and it is believed that the Earth's atmosphere is responsible for the extent of the enlargement, but it is realized that the atmospheric absorption cannot explain light absorption at a height as high as $90 \mathrm{~km}$ above the Earth, as required by this hypothesis. It was also argued that the irradiation of the Moon in the Earth's shadow during the eclipse is caused by the refraction of sunlight in the upper regions of the Earth's atmosphere. However, the shade toward the center is too bright to be accounted for by refraction of visible sunlight. Although these assumptions are not trifling, we attribute the majority of these abnormalities to the Allais eclipse effect. This effect would cause a slight decrease of gravity during the eclipse: the geodesics would be displaced a small amount outwards; the ray of light coming from the Sun, passing close by the Moon would be less attracted, which would expand the shadow cone of the Moon. On the other hand, the rays emanating from the Moon would have a shorter wavelength and therefore the luminescence would increase by anti-Stoke Raman effect: the scattered photon has more energy than the absorbed photon.
\end{abstract}

\section{Keywords}

Lunar Eclipses, Enlargement of the Earth's Shadow, Luminescence, Allais Eclipse Effect, Anti-Stokes Raman Effect

\section{Introduction}

We know that astronomical data give us accurate values of the radii of the Sun, the Earth and the Moon. Furthermore, the knowledge of their relative distances predicts quite accurately the instant when the umbra-penumbra limit sweeps 
some specific craters on the Moon during lunar eclipses. Since the 1830s, crater timing has been used during lunar eclipses to measure the length of the Earth's shadow. The method is simple: one takes the timing of lunar features (craters, limbs, ridges, peaks, bright spots) as they enter and exit the umbra. The SunEarth-Moon geometry being known quite precisely is then possible to calculate the size and shape of the Earth's umbra at the Moon. Measurements that vary from one eclipse to the next can now be made with low-power telescopes or a clock synchronized with radio time signals. However, it has systematically been found that the shadow of the Earth seems to be $2 \%$ larger than what is expected from geometrical predictions.

Even, if it is believed that the thickness of the Earth atmosphere is responsible for that displacement [1], it was realized that the atmospheric absorption cannot explain the absorption of light at a height of up to $90 \mathrm{~km}$ above the Earth, as required by this hypothesis. It may be noted in particular that Link [2] has firmly established a relationship between the enlargement of the Earth's shadow during lunar eclipses and the presence of meteors, which have the ability to distort the optical properties of the atmosphere when they are braked at high altitudes [3].

It has been said that the pronounced red colour in the inner portions of the umbra during an eclipse of the Moon is caused by refraction of sunlight through the upper regions of the Earth's atmosphere, but the umbral shadow towards the centre is too bright to be accounted for by refraction of visible sunlight.

In Sections 2 and 3, we give a brief history of the enlargement of the Earth's umbra and the excess of light into the Earth's shadow onto the Moon during lunar eclipses. We present some accepted interpretations and we show how the Allais effect, which occurs at the time when problems arise related to these anomalies, leads us to reject these interpretations. In Section 4, it emerges from a discussion that, failing to have an answer that would explain the two coexisting anomalies, the Allais eclipse effect currently remains the only viable option. Experiments are proposed as much to corroborate the observations of the two anomalies as to test the Allais eclipse effect. We conclude that both anomalies during lunar eclipses are caused by a lunar Allais effect.

\section{Enlargement of the Earth's Umbra}

\subsection{Brief History of the Enlargement of the Earth's Umbra on the Moon during Lunar Eclipses}

In the early 1700s, Philippe de La Hire made a curious observation about Earth's umbra. The predicted radius of the shadow needed to be enlarged by about 1/41 in order to fit timings made during an eclipse of the Moon (La Hire 1707). Additional observations over the next two centuries revealed that the shadow enlargement was somewhat variable from one eclipse to the next [4].

Chauvenet (1891) adopted a value of $1 / 50$, which has become the standard enlargement factor for lunar eclipse predictions published by many national in- 
stitutes worldwide. Some authorities dispute Chauvenet's shadow enlargement convention [5]. Danjon (1951) notes that the only reasonable way of accounting for a layer of opaque air surrounding Earth is to increase the planet's radius by the altitude of the layer [6]. This can be accomplished by proportionally increasing the parallax of the Moon. The radii of the umbral and penumbral shadows are then subject to the same absolute correction and not the same relative correction employed in the traditional Chauvenet 1/50 convention. Danjon estimates the thickness of the occulting layer to be $75 \mathrm{~km}$ and this results in an enlargement of Earth's radius and the Moon's parallax of about 1/85. Since 1951, the French almanac Connaissance des Temps has adopted Danjon's method for the enlargement Earth's shadows in their eclipse predictions

Danjon's method correctly models the geometric relationship between an enlargement of Earth's radius and the corresponding increase in the size of its shadows. Meeus and Mucke (1979), and Espenak (2006), both use Danjons method. However, the resulting umbral and penumbral eclipse magnitudes are smaller by approximately 0.006 and 0.026 respectively as compared to predictions using the traditional Chauvenet convention of $1 / 50$.

For his part, in an analysis of 57 eclipses over a period of 150 years, Link (1969) found an enlargement of the shadow of $2.3 \%$ on average. Furthermore, schedules inputs and outputs of the crater through the umbra for four lunar eclipses from 1972 to 1982 strongly support the Chauvenet value of $2 \%$. Of course, the small magnitude difference between the two methods is difficult to observe because the edge of the umbral shadow is diffuse. From a physical point of view, there is no well defined border between the umbra and the penumbra. The shadow density actually varies continuously as a function of radial distance from the central axis out to the extreme limit of the penumbral shadow. However, the density variation is most rapid near the theoretical edge of the umbra. Kuhl's (1928) contrast theory demonstrates that the verge of the umbra is perceived at the point of inflexion in the shadow density. This point appears to be equivalent to a layer in Earth's atmosphere at an altitude of about 120 to $150 \mathrm{~km}$. The net enlargement of Earth's radius of $1.9 \%$ to $2.4 \%$ corresponds to an extension of the umbra of $1.5 \%$, to $1.9 \%$, in reasonably good agreement with the conventional value.

It seems that the increase of the Earth's umbral shadow during eclipses of the Moon is the classical value of $2 \%$ (the rule of the fiftieth) used in most calculations of lunar eclipses [7].

\subsection{Accepted Interpretation of the Enlargement of the Umbra}

Numerous reports show that the umbra-penumbra limit appears significantly displaced on the moon during an eclipse. It is believed that the thickness of the Earth atmosphere is responsible for that displacement [8] [9] [10]. In order to study more deeply the phenomenon showing that the umbra-penumbra limit appears significantly displaced on the Moon during an eclipse, it is important to 
evaluate if the reported increase of $2 \%$ of the Earth's shadow on the Moon corresponds to a reasonable value of the height at which the atmosphere is opaque. Calculations indicate that this enlargement corresponds to a terrestrial altitude of $92 \mathrm{~km}$.

This usual interpretation of the umbral enlargement forces us to believe that the atmosphere is normally opaque up to $92 \mathrm{~km}$ or so. But how is this possible when, at this altitude, the air is extremely rarefied? It is the altitude close to the orbit on which a satellite travels around the Earth.

In fact, according to data [11], the atmospheric pressure at $90 \mathrm{~km}$ above sea level is about half a million times smaller than that at sea level. Above $15 \mathrm{~km}$, the atmosphere becomes relatively transparent to light, since $90 \%$ of the air and almost all the humidity and pollution are below that level. That makes an enlarged obscuration due to the opacity of the atmosphere of only $0.3 \%$ which is much smaller than the $2.0 \%$ reported.

Furthermore, the eruption of volcanos cannot explain the larger shadow. According to some, the altitude reached by some material ejected from volcano El Chichon is in the stratosphere, some 26 kilometers (16 miles) above Earth's surface - roughly $50 \%$ higher than material from the famous Mount St. Helens [12]. Since the atmosphere does not appear to be responsible for the umbra-penumbra limit displacement of $2 \%$ on the Moon, then what is the cause?

F. Link argues that the meteoric dust in the upper atmosphere of the Earth is at the origin of the additional weakening of the light and the expansion of the Earth's darkness [13] [14]. We might point out, in particular, that Link has actually established a concomitance between the enlargement of the Earth's umbra during lunar eclipses and the presence of meteors, which are capable of distorting the optical properties of the atmosphere when they are decelerated at high elevations [3] [15].

Paul Marmet and Christine Couture [1], for their part, believe that the actual umbra of the Earth projected on the Moon is not as big as observed, that the sensitivity of the eyes is a factor leading necessarily to an umbral enlargement and that almost the totality of the reported umbra-penumbra limit displacement is an optical effect that has nothing to do with the thickness of the Earth atmosphere.

For our part, we believe that the observed times to browse the path of the Moon through the Earth's obscurity deviate from the predicted times and that some variations in colour, size and shape of the umbra occur in the darkness. We attribute this deviations and variations to the Allais eclipse effect.

\subsection{Umbral Enlargement and the Allais Eclipse Effect}

During an eclipse of the Moon, it is predicted geometrically that the photons from the Sun describe a rectilinear trajectory as if they were little deflected and pass at a minimum approach distance $R_{E}^{\prime}$ from the centre of the Earth (slightly larger than the radius $R_{E}$ of the Earth), before moving to the Moon. A ray of 
sunlight passes close to the Moon at point $R_{M}^{\prime}$ of minimum approach to arrive at the point $P$ at the end of the shadow cone of the Earth. The trajectory followed by the solar photons shapes the curvature of minimum approach of the Earth and the Moon.

However, we have serious reasons to believe that during a lunar eclipse, with the Earth interposed between the Moon and the Sun, there would be a kind of anti-gravity on the Moon which would be manifested by a deviation of light. This is precisely the Allais effect [16] [17] [18].

The orbital radius seems longer, which means that the curvature of minimum approach of the Earth and the Moon is shifted outward. Since the curvature is the inverse of the square of the radius, the curvature is even smaller than the radius is large. This is grounded in the Newtonian logic stating that gravity identified to the curvature is all the more weak as the orbital radius is large. The deviated photons will pass at a distance of minimum approach $R_{E}^{\prime \prime}\left(R_{E}^{\prime \prime}=R_{E}+\Delta R_{E}\right)$ from the centre of the Earth and at the point $R_{M}^{\prime \prime}\left(R_{M}^{\prime \prime}=R_{M}+\Delta R_{M}\right)$ of minimum approach of the Moon to end at the point $P^{\prime \prime}\left(P^{\prime \prime}=P+\Delta P\right)$, casting an enlarged umbra cone. It matches with the observed cone of the enlargement of the Earth's umbra.

During a lunar eclipse, it is predicted geometrically that the photons from the Sun describe a rectilinear trajectory as if they were a little deflected (Figure 1).

Inevitably, sunlight, observed on the eclipsed Moon, will tend to move away. The photon has an "inertial mass" equal to $h v / c^{2}$ equivalent to a "gravitational mass" also equal to $h v / c^{2}$. The energy of the "fallen" photon from the Sun will be $m\left(g-g^{\prime}\right) H$ instead of $m g H$ ( $m=$ inertial and gravitational mass of the photon; $g=$ acceleration due to gravity, $H=$ height). The gravitational mass of photons, lessened, takes distance, so increasing the darkness.

We are witnessing an abnormal gravitational frequency shift.

Suppose that in normal times the light is emitted by the Sun at the height $H$ (distance Sun-moon) [19]. The total energy of a photon of frequency $V$ and

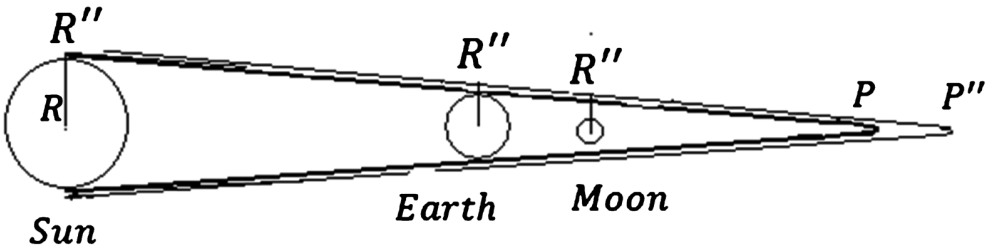

Figure 1. Exaggerated diagram (for comprehension) of the cones of the umbra. The illumination of the Earth by the Sun projects into space a converging cone of umbra and a divergent cone of penumbra whose conical generators are tangent to the Earth and the Sun. We represent here that the converging cone of umbra to illustrate the enlargement of the earth's shadow. The umbra cone does not completely obscure the Moon and, as early as the $18^{\text {th }}$ century, astronomers knew that the shadow limit was a little further $\left(P^{\prime \prime}\right)$ than according to the geometric path of the rays $(P)$. The quasi-parallel interior tangents to the Sun and the Earth give the two interior cones, predicted and observed, of the umbra. The tangent $R_{S}^{\prime \prime} R_{E}^{\prime \prime} R_{M}^{\prime \prime} P^{\prime \prime}$ gives the observed cone of the umbra while the tangent $R_{S} R_{E} R_{M} P$ gives the calculated cone of the umbra. 
energy $h v$, reaching the lunar surface has become

$$
h v^{\prime}=h v+h v g H / c^{2}
$$

The receiver, placed on the lunar ground, detects a frequency $v^{\prime}$ greater than $v$ of the solar source ( $g$ designates the lunar gravitational field):

$$
v^{\prime}=v\left[1+g H / c^{2}\right] .
$$

During a lunar eclipse, due to a potential loss of attraction, the lunar gravitational field $g$ amounts to $g-g^{\prime}$. When a photon emitted by the Sun reaches the surface of the Moon, he lost potential energy $\left(h v / c^{2}\right)\left(g-g^{\prime}\right) H$ and won the kinetic energy $\left(h v / c^{2}\right)\left(g-g^{\prime}\right) H$. Its total energy has become

$$
h\left(v^{\prime}-v^{\prime \prime}\right)=h v+\left(h v / c^{2}\right)\left(g-g^{\prime}\right) H .
$$

The frequency $v^{\prime}-v^{\prime \prime}$ of the photon at its arrival at the surface of the eclipsed Moon is less red-shifted relative to its initial frequency, according to the relation

$$
v^{\prime}-v^{\prime \prime}=v\left[1+\left(g-g^{\prime}\right) H / c^{2}\right] .
$$

The receiver detects a frequency $v^{\prime}-v^{\prime \prime}$ slightly smaller than $v^{\prime}$ of not eclipsed Moon. This means a small blue shift for the Sun during the eclipse.

If this hypothesis is correct which consists to declare that the Allais effect causes a kind of repulsion between the three celestial bodies involved, there should be a variation of the gravitational potential. This means that the gravitation will influence the geometry of space-time: the time of clocks and the length measured by a rule will be affected depending on whether there is more or less gravity. Einstein's general theory of relativity predicts that a clock in the presence of weak gravity runs more rapidly than one located where gravity is stronger. Consequently, the frequencies of radiation emitted by atoms in the presence of a weak gravitational field are shifted to higher frequencies when compared with the same emissions in the presence of a strong field. The light of the Sun observed on an eclipsed Moon should be blue shifted; a fraction of the solar gravitational redshift $\left(g^{\prime} R / c^{2}\right)$ which is about two parts in a million [20].

The widening of the Earth's shadow on the Moon would not be due to a greater density of the upper atmosphere of the Earth, which would make it as opaque as the lower atmosphere, it would be caused by a gravitational potential temporarily decreased. An "alleviated" matter would dictate to space-time a smaller degree of curvature; the space-time would in turn impose to matter to move on a larger orbital radius.

\section{Excess of Light into the Earth's Shadow}

\subsection{Brief History of the Excess of Light into the Earth's Shadow on the Moon during Lunar Eclipses}

The first work on the variation in brightness of eclipses was executed by André-Louis Danjon in 1920. He devised a scale of brightness for total lunar eclipses, from 0 for invisible to 4 for very brilliant. He used it to analyze data on 
eclipses extending back over three and a half centuries, and showed a correlation between the eclipse brightness and the solar activity. But a series of three-color photometric observations of Moon eclipse, made by him and his associates between 1932 and 1957, appears to show a clear correlation between the eclipse brightness and the geomagnetic planetary index $K_{p}[21]$. So the new data seems to contradict the first Danjon's conclusion.

An attempt to interpret the relation with $K_{p}$ in terms of lunar luminescence indicates that the change in the eclipse brightness is in accord with the rate of increase of the plasma energy, as predicted by the experiment of Snyder et al. (1963) [22]. However, there is some difficulty in the required proton density being greater than the observed value by an order of magnitude. The same calculations show that luminescence to be visible in ordinary moonlight requires a plasma energy at least three orders of magnitude greater than the maximum value predicted by Snyder et al. They also show that the reported dates of these observations fall on geophysically quiet days, as well as on dates of high $K_{p}$. The above conclusion agrees with the result of calculations by Ney et al. in 1966.

On the other hand, J. Dubois and F. Link in 1969 found a correlation between the brightness of the eclipsed Moon and the solar activity, as had been suggested by Danjon on the basis of its first observations. They demonstrated that the brightness of eclipse was related not only to the sunspots number but also to the height of the latitude. They showed an annual correlation between the heliographic latitude of the apparent centre of the Sun's disk and eclipse brightness [23] [24].

It was suggested that the brightness anomaly of the umbral region during an eclipse of the Moon would be caused by refraction of sunlight through the upper regions of the Earth's atmosphere. The red coloring arises because, they say, sunlight reaching the Moon must pass through a long and dense layer of the Earth's atmosphere, where it is scattered. Shorter wavelengths are more likely to be scattered by the small particles and so, by the time the light has passed through the atmosphere, the longer wavelengths dominate. This resulting light we perceive as red. The amount of refracted light depends on the amount of dust or clouds in the atmosphere; this also controls how much light is scattered. In general, the dustier the atmosphere, the more that other wavelengths of light will be removed (compared to red light), leaving the resulting light a deeper red color [25].

Despite this reasoning, it has been found that towards the centre the umbra is too bright to be accounted for by refraction of visible sunlight. F. Link proposed that this excess be interpreted as luminescence [26]. He concluded that about 10 percent of the Moon's optical radiation is caused by luminescence. Observations seem to confirm the existence of lunar luminescence. The term luminescence can be applied to any object that emits light in addition to the usual reflected light [27]. The main characteristic of luminescence is that the emitted light is an attribute of the object itself, and the light emission is stimulated by some internal 
or external process. As external process, Link suggested the luminescence of the lunar surface by X-ray bombardment from the uneclipsed regions of the solar corona, as suggested by Link. This theory is supported by the variation of a factor of 100 , between solar maximum and minimum, of the intensity of certain wavelengths of X-rays [28].

Another possible mechanism by which the eclipsed Moon shines results from the fact that the Moon is covered by a fine layer of meteoric dust, and would therefore contain quantities of the achondritic enstatites. This type of stony meteorite produces the luminescence when protons and electrons of the solar wind are deflected and impinge on the lunar surface during a total eclipse. An experiment performed by Zdenek Kopal, C. J. Derham and J. E. Geakel in 1963 showed that certain meteorite specimens glowed with a strange red light, same colour as the umbra in eclipse, when bombarded by high energy protons in the laboratory [24].

It appears to us that the excess of irradiation of the Moon in the shadow of the Earth during the eclipse is partially caused by refraction in the atmosphere, but that it prevailingly depends of the light emission stimulated by an internal process linked to the Allais eclipse effect.

\subsection{Luminescence of the Eclipsed Moon and Allais Effect}

The time of vibration $(T)$ of atoms and molecules of luminescent gases in the fieldless region of space is $T=\tau$ ( $T$ is the time of vibration in the atom at rest; $\tau$ is the modified time of vibration) [29]. In a region of space with the gravitational field, the time of vibration is altered to

$$
\tau=T /(1-\gamma / 2)=T /\left(1-v^{2} / c^{2}\right)=T /\left(1-\phi / c^{2}\right)
$$

[ $\gamma$ contains the gravitational potential $\phi$ $\left.\left(\gamma=2 G M / R c^{2}=2 v^{2} / c^{2}=2 \phi / c^{2}\right)\right]$.

Our assumption is that at the surface of an eclipsed Moon there is a weaker gravitational field than in a frame of reference without eclipse $\left(\gamma^{\prime}<\gamma\right)$

$$
\begin{gathered}
{\left[\tau=T /\left(1-\gamma^{\prime} / 2\right)\right]<[\tau=T /(1-\gamma / 2)]} \\
\text { with eclipse without eclipse }
\end{gathered}
$$

During the lunar eclipse time, the gravitational potential $\phi$ of the Moon is conjecturally diminished; the time $\tau$ of the vibration of the atom is shorter. The metric of the obscured Moon is affected and the ticking of time accelerates relative to the system without eclipse in which the atom is considered at rest.

Consequently, the red shift of the spectral lines of light that comes from the layer of particles on the ground will have a small additional blue "Allais" shift which reduces the "Einstein" redshift [19]. As the Einstein effect (i.e. the tiny frequency shift of spectral lines in a gravitational field) is directed towards the blue, there is thus more internal electromagnetic energy. It appears that this blueshift by variation in the reduction of the mass could be a form of atomic excitement at the level of electrons, as Brownian motion. More specifically, we 
would say that the Allais eclipse effect would have engendered a significant change of wavelength within the molecules of matter [30]. The excess of luminescence would be the imprint left on the light by the intramolecular oscillation of the atoms constituting the molecules of the lunar soil which spreads it. A Raman effect caused by an Allais effect, in some way.

\subsection{An Anti-Stoke Raman Effect Induced by an Allais Effect}

We assume that the lunar gravitational potential $\phi$ can be reduced in times of eclipse, what would accelerate the vibration of atoms.

A molecule can be excited to a very high energy state. The amount of energy necessary to reach this excited state is $h v_{o}$. Therefore, the relaxation of the molecule to the ground-state vibrational energy level $v=0$ results in the emission of a photon of energy $h v_{o}$. This emission is usually observed in the visible spectral region and is called Rayleigh scattering. We think that the rapid oscillation of a light wave passing by the intramolecular level of atoms which constitute the molecules diffused by the lunar soil could be similar to an effect Raman anti-Stoke [31].

The scattering of light on the optical modes is designated Raman effect. It is different from the Rayleigh scattering because the scattered light changes the frequency of the spectrum active vibration. Historically, the effect was first observed with molecules. Molecules vibrate, and each molecular oscillation corresponds to a certain amount of energy. In the scattering process, this energy is added or subtracted from the incident light. An anti-Stokes Raman effect occurs when the molecule absorbs an incident light of frequency $v_{o}$ and reemits it at a higher frequency.

Thus, during the eclipse of the Moon, the excited molecule would oscillate from a superior vibrational energy level, say $v=1$. The energy absorbed in this process is still $h v_{o}$. The molecule can relax to the original $v=1$ vibration energy level and emits a photon $h v_{o}$; however, the relaxation can be to the ground state. The return to the state $v=0$ results in the emission of a photon which is $h v_{1}$ greater than the exciting energy $h v_{o}$ from level 1 . The photon energy emitted is $h\left(v_{o}+v_{1}\right)$. Spectral lines with frequencies higher than $v_{o}$ are labeled anti-Stokes lines [32]. This Raman shift induces a brighter electromagnetic radiation.

\section{Discussion and Conclusion}

In 2009, NASA's Lunar Reconnaissance Orbiter (LRO) was launched with the Lunar Crater Observation and Sensing Satellite (LCROSS) on the first U.S. mission to the Moon in over 10 years. LRO gathered information on day-night temperature maps, contributed data for a global geodetic grid, and conducted high-resolution imaging. During lunar eclipses, the solar-powered orbiter also falls in Earth's shadow, cutting it off from the source of its power. The mission controllers can then use an instrument-called Diviner-that can watch how the 
lunar surface responds to the rapid change in temperature caused by a lunar eclipse. These data which help scientists better understand the composition and properties of the surface could be a scientific boon for understanding both anomalies [33] [34] [35].

We believe that almost the totality of the reported umbra-penumbra boundary shift and the excessive clarity of the penumbra reveal a lunar Allais effect on the Moon's shadow that has nothing to do with the thickness of the Earth's atmosphere. Both phenomena were reported during each and every lunar eclipse recorded for the past 180 years. They occur during lunar eclipses and are correlated. Dr Marmet demonstrated that the Earth's atmosphere cannot be the cause of the enlargement of the Earth's shadow. He concludes that it is an optical illusion, but neither addresses nor explains the second offset: the excessive brightness of the penumbra [1]. If he is right to say that the Earth's atmosphere is not responsible for the $2 \%$ umbra-penumbra limit shift on the Moon, he is wrong to evoke the optical illusion. NASA records anomalies without providing an explanation, the priorities being elsewhere. The door is open to researchers to probe the reasons and suggest fields to explore. The list of our references shows that they cannot be explained by current science, which leaves only one option: the lunar Allais effect.

But the bottleneck, which means that this aspect of science remains speculative even as Professor Allais' experiments have validated the solar eclipse effect, is the question of a lack of willingness to experiment. How can interest be aroused in experimenters for whom the scientific value of precise experience is dependent on their theoretical interpretation? Classical conservative physical thought cannot tolerate the defeat of the current theory of gravitation when applied to the case of the influence of the attraction of the Sun and the Moon on the motion of the paraconic pendulum, whether these are the amplitudes of the lunisolar periodic components or the anomalies observed during the total eclipses of the Sun [16].

However, the most accommodating physicists say they do not rely on the more or less contradictory experiments carried out so far; they would like experiments operated with a paraconic pendulum at any point similar to the pendulum used by M. Allais, or they would like to turn to more radical experiments, like those intended for modern theories. For example, the atomic clock cooled by cesium laser (PHARAO) [36] placed by the European Space Agency (ESA) on the International Space Station (ISS) could have been used. In default of confirm doubtful fashionable theories, this high technology could test the Allais effect and supply the way to tie, by a new theoretical link, the facts observed during the eclipses to the physical laws having received the sanction of a rigorous experimental control.

In conclusion, it seems that two noticed anomalies during lunar eclipses, the enlargement of the Earth's shadow delineated onto its satellite and an excessive illumination of the penumbra, adjusted ad hoc to the Earth's atmosphere, would rather be caused by an Allais eclipse effect, i.e., a repulsion that occurs when the 
Moon passes directly behind the Earth into its umbra, when Sun, Earth and Moon are closely aligned in space. This is consistent with our knowledge of the solar eclipse, with the calculation of the abnormal spontaneous acceleration of the Moon during the solar eclipse in June 1954 (paraconical pendulum of Maurice Allais) and the result recorded by a gravimeter during the solar eclipse of 1997.

(To know more about lunar eclipses by pictures, references [37] [38] [39] [40] have been added).

\section{Conflicts of Interest}

The author declares no conflicts of interest regarding the publication of this paper.

\section{References}

[1] Marmet, P. and Couture, C. (1997-99) Enlargement of the Earth's Shadow on the Moon: An Optical Illusion. Physics Department, University of Ottawa, Ottawa.

[2] Link, F. (1970) La Lune, Collection Science d'Aujourd'hui. Albin Michel, Paris, 107.

[3] Link, F. and Linkova, Z. (1954) Agrandissement de l'ombre terrestre pendant les éclipses de Lune. Institut astronomique, Ondrerov.

[4] Espenak, F. and Meeus, J. (2009) Enlargement of Earth's Shadows. NASA, Washington DC.

[5] Chauvenet, W.A. (1891) Manual of Spherical and Practical Astronomy, Vol. 1. (1960) Dover Reprint, New York.

[6] Danjon, A. (1951) L'Astronomie, 65, 51-53.

[7] Meeus, J. (1972) Ciel et Terre, 88, 491.

[8] Sinnott, R.W. (1983) Sky \& Telescope, Readers Gauge the Umbra Again. 387.

[9] Sinnott, R.W. (1992) Sky \& Telescope, a Tale of Two Eclipses. 678.

[10] Soulsby, B.W. (1984) Journal of the British Astronomical Association, Lunar Eclipse Crater Timing Programme, 95, 16-21.

[11] Lang, K.R. (1992) Astrophysical Data: Planets and Stars. Springer-Verlag, New York, 36.

[12] McCormick, P. (1982) Sky \& Telescope. 390.

[13] Link, F. (1953) Sur la grandeur des poussières météoriques dans l'atmosphère terrestre. Publishing House of the Czechoslovak Academy of Sciences. NASA Astrophysics Data System.

[14] Link, F. and Linková, Z. (1954) Agrandissement de l'ombre terrestre pendant les éclipses de Lune; influences météoriques. Publishing House of the Czechoslovak Academy of Sciences. Provided by the NASA Astrophysics Data System.

[15] Fesenkov, V.G. (1970) Astronomicheskii Zhurnal, 47, 237-245.

[16] Allais, M. (1997) L’Anisotropie de l'Espace. Edition Clément Juglar, Paris, 200.

[17] Bagdoo, R. (2009) Journal of Modern Physics, 11, 1620-1638.

https://doi.org/10.4236/jmp.2020.1110101

[18] Bagdoo, R. (2010) Is the Growth of the Astronomical Unit Caused by the Allais Eclipse Effect? ViXra, General Science Journal, Internet Archive, Issuu, Scribd.

[19] Mavridès, S. (1988) La Relativité, Que sais-je? Presses universitaires de France, 
Paris, 105-106, 112, 119.

[20] Schwinger, J. (2002) Einstein's Legacy. Dover Publication, Inc., Mineola, 146.

[21] Matsushima, S. (1966) The Astronomical Journal, 71, 699-705. https://doi.org/10.1086/110174

[22] Snyder, C.W., Neugebauer, M. and Rao, U.R. (1963) Journal of Geophysical Research, 68, 6361-6370. https://doi.org/10.1029/JZ068i024p06361

[23] Dubois, J. and Link, F. (1970) Analyse photométrique de l’ombre intérieure pendant les éclipses de Lune. Publishing House of the Czechoslovak Academy of Sciences. Provided by the NASA Astrophysics Data System.

[24] Shepherd, J.S. (1982) Journal of the British Astronomical Association, 92, 66-67.

[25] Wikipedia. Lunar Eclipse. https://en.wikipedia.org/wiki/Lunar eclipse

[26] Link, F. (1959) Densité de l'ombre pendant les éclipses de Lune. Publishing House of the Czechoslovak Academy of Sciences. Provided by the NASA Astrophysics Data System.

[27] Sandulak, N. (1964) Jurgen Stock, Indication of Luminescence Found in the December 1964 Lunar Eclipse. Cerro Tololo Inter-American Observatory, La Serena, 237. (1965) Provided by the NASA Astrophysics Data System.

[28] Jamieson, D.N. (1995) The July Lectures in Physics-1995 Light without Heat: Luminescence in Moonlight. School of Physics, University of Melbourne, Melbourne.

[29] Born, M. (1962) Einstein's Theory of Relativity. Dover Publications, Inc., Mineola, 352-353.

[30] Radounskaïa, I. (1972) Idées folles. Édition MIR, Moscou, 68-69, 78-82.

[31] Lerner, R.G. and Trigg, G.L. (1990) Raman Spectroscopy, Encyclopaedia of Physics. VCH Publishers, Inc., Hoboken, 1034.

[32] Möller, K.D. (1988) Optics. University Science Books, Sausalito, 574-5, 621.

[33] Earth's Moon. https://solarsystem.nasa.gov/moons/earths-moon/lunar-phases-and-eclipses

[34] Lunar Reconnaissance Orbiter. https://solarsystem.nasa.gov/missions/lro/in-depth

[35] NASA (2011) Need to Know: Lunar Eclipse and LRO. YouTube, NASA Goddard.

[36] Bagdoo, R. (2012) The PHARAO/ACES Mission and the Allais Effect. General Science Journal, ViXra, Issuu, Scribd.

http://gsjournal.net/Science-Journals/Research\%20Papers/View/4292

[37] Jones, T. (2011) Total Lunar Eclipse 10th December 2011. https://communicatescience.com/zoonomian/2011/12/10/total-lunar-eclipse-10th-d ecember-2011

[38] Google, 8477 Lunar Eclipse Premium High Res Photos, Getty Images.

[39] Google, Images correspondant à Lunar eclipses.

[40] NASA (2014) Understanding Lunar Eclipses. YouTube. 